\title{
RFID Passive Gas Sensor Integrating Carbon Nanotubes
}

\author{
Cecilia Occhiuzzi, Member, IEEE, Amin Rida, Member, IEEE, \\ Gaetano Marrocco, and Manos Tentzeris, Fellow, IEEE
}

\begin{abstract}
Carbon nanotube (CNT) composites are sensitive to the presence of gases due to their high surface-to-volume ratio and hollow structure that are well suited for gas molecule absorption and storage. Such sensing capability is here integrated with UHF RF identification (RFID) technology to achieve passive and lowcost sensors, remotely readable. CNT film (buckypaper) is used as a localized variable resistive load integrated into a tag antenna, which becomes able to transduce the presence of hazardous gas in the environment, ammonia in this case, into a change of its electromagnetic features. The dynamic range and the hysteresis of the radio sensor are investigated by simulations, equivalent circuits, and articulated experimentations within a true RFID link, providing the proof of concept and some guidelines for tag design.
\end{abstract}

Index Terms-Nanotube, passive gas sensor, RF identification (RFID).

\section{INTRODUCTION}

$\mathbf{R}$ ECENTLY, there has been increasing effort toward combining the capabilities of sensors and wireless devices in order to collect, process, and transmit time-varying information about objects/personnel. RF identification (RFID) passive sensing has been successfully explored as a low-cost candidate toward lightweight, reliable, energy-efficient, and even batteryless durable wireless sensing devices. Proposed examples, already theoretically investigated or experimentally tested, include: temperature sensing [1], [2], solar powered and piezoscavenging batteryless wireless sensor tags [3], human monitoring by means of implanted devices [4], biochemical sensors [5], strain sensor [6], [7], motion sensor [8], moisture [9], and gas detection [10]. However, there is still significant work to do to fully master the cohabitation and integration of sensing and communication capabilities [11].

One of the most attractive applications of wireless sensors is the remote monitoring of environments to detect the presence of hazardous gases within industrial sites (supply chain and food integrity) or to control the quality of work spaces. Traditional

Manuscript received February 17, 2011; revised June 26, 2011; accepted July 08, 2011. Date of publication August 30, 2011; date of current version October 12, 2011. This work was supported by the National Science Foundation (NSF), Interconnect Focus Center/Semiconductor Research Corporation (IFC/SRC), and New Energy and Industrial Technology Development Organization (NEDO)-Japan, and by the Italian Ministry of University under Project PRIN2008 "RFID MULTI-TAG."

C. Occhiuzzi and G. Marrocco are with the Dipartimento di Informatica, Sistemi e Produzione (DISP), University of Rome "Tor Vergata," Rome 00133, Italy (e-mail: occhiuzzi@disp.uniroma2.it; marrocco@disp.uniroma2.it).

A. Rida and M. Tentzeris are with the Department of Electrical and Computer Engineering, George Institute of Technology, Atlanta, GA 30332 USA (e-mail: arida@gatech.edu; emmanouil.tentzeris@ece.gatech.edu).

Digital Object Identifier 10.1109/TMTT.2011.2163416 gas-sensing mechanisms exploit vapor-sensitive polymers, semiconductor metal oxides, or porous structures as sensitive material, which require microfabrication techniques, power supply, and data-processing electronics that may preclude many low-cost monitoring applications [12]. Carbon nanotube (CNT) composites [13] have been recently found to perform as a gas sensor due to their high surface-to-volume ratio and hollow structure that are well suited for gas molecules absorption and storage. Upon exposure to small quantities of certain gases, CNTs change their physical properties, such as their electrical conductance (molecules act as electron donors or acceptors), and this variation can be easily detected by various methods [14]. Concerning wireless systems, for example, a CNT-based inductor-capacitor resonant circuit $(L C)$ with a multiwall CNT-SiO2 composite as a sensing layer has been proposed in [15] as a passive wireless gas sensor. The presence of gas in the air produces a change of the dielectric properties of the CNT coating, and hence of the resonant frequency of the system, detectable by a loop antenna located approximately $15 \mathrm{~cm}$ from the sensor. However, the extremely low frequency $(\sim 10 \mathrm{MHz})$ and the magnetic coupling between the radiating structures make the proposed platform not suitable for medium/long-range applications. The same sensing approach has been evaluated also in [16] for a wired configuration.

From a different side, several CNT-based antennas have also been developed for their attractive characteristics. Due to the very high inductance per unit length [17], it is possible to fabricate CNT nanoantennas, while their relatively low cost, wide bandwidth, and simplicity of fabrication can indeed offer great advantages in RFID applications [18]. Finally, the good mechanical properties of this kind of material, such as flexibility, strength, and low weight, suggests interesting opportunities in wearable electronics and antennas [19].

While the idea to integrate sensing carbon nanostructures into a passive RFID tag was first introduced in [10], no attempt has been done up to date to design, fabricate, and test a complete CNT-tag working as a medium-range passive radio sensor.

In this context, this work is aimed to theoretically and experimentally demonstrate for the first time the feasibility of a fully working RFID sensor tag doped with single-wall carbon nanotubes (SWCNTs) sensitive to the presence of ammonia gas and to characterize its true sensing capabilities and limitations. The physical rationale of the proposed sensor lies in the clear dependence of the tag's radiation performances on the physical and chemical features of the integrated CNT, strongly affected by the close surrounding environment (Fig. 1). When the environment where the tag is placed undergoes changes in time, due to the presence of ammonia or other gases, the CNT's electrical 


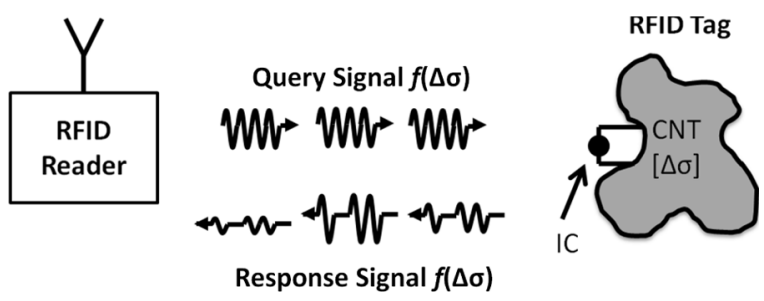

Fig. 1. Change of the electromagnetic response of RFID gas sensor can be related to a variation of the features of the integrated CNT, e.g., its conductivity $\Delta \sigma$

features (mainly the conductivity $\sigma$ ) accordingly change. These variations affect the tag's performance and can be remotely detected by the reader [20].

This paper is organized as follows. Section II will be focused on the fabrication and characterization of the SWCNT buckypaper, to be used as part of the radio-sensor. The RFID sensing paradigm, as well as its basic equations, are then recalled in Section III with particular attention to the definition of useful sensing identifiers, which can be easily extracted from raw measured data. The design of the RFID passive radio sensor is then discussed in Section IV by means of equivalent circuit model and experimentations. Finally, in Section V, the sensing features of the CNT-based RFID tag are fully investigated in the American and European UHF band by means of many experimental tests.

\section{SWCNT MATERIAL PREPARATION AND CHARACTERIZATION}

Several deposition techniques have been proposed for CNTbased antennas and electronics: inkjet and screen-printing procedures [10], chemical vapor deposition (CVD) processes [19], and thin films [18] are recent examples of useful techiques able to offer high conductive CNT samples.

SWCNT film, also referred to as buckypaper, offers isotropic conductivity, good mechanical strength and flexibility and gives the possibility to easily cut out the shape of the desired antenna [21]. The fabrication procedure generally consists of filtering SWCNT suspension in acqueous or organic media, such as Triton $\mathrm{X}-100$ or dimethyl formamide (DMF), under positive or negative pressure.

In this work, an alternative preparation method is presented, where the filtration over membrane has been replaced by evaporation in a controlled environment. The process is schematically shown in Fig. 2. A 100-mg purified high functionality with carbonaceous purity $\geq 90 \%$ SWCNT powder from Carbon Solutions Inc., Riverside, CA, has been dispersed in $66 \mathrm{~mL}$ of water by sonication at $30 \mathrm{~W}$ for $60 \mathrm{~min}$. Next, in order to get rid of the water and form the CNT buckypaper, the dispersion has undergone an overnight evaporation at $70^{\circ} \mathrm{C}$. Finally, a circular sheet with diameter $d=90 \mathrm{~mm}$ has been produced over a polyamide membrane. The thickness of the buckypaper is determined by the total amount of CNTs used in the dispersion per unit surface of the membrane since the water is removed during the evaporation process. It can be evaluated in first approximation by a simple mathematical proportion with the density and thickness achieved in [22]. With a calculated density

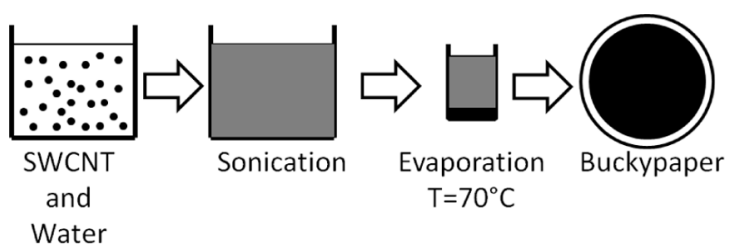

Fig. 2. Buckypaper preparation steps.

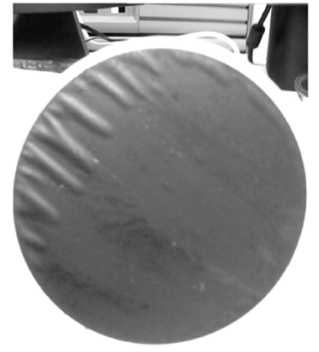

a)

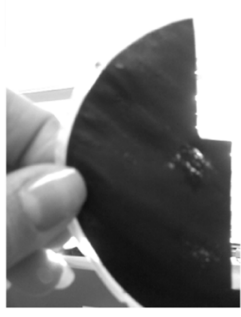

b)

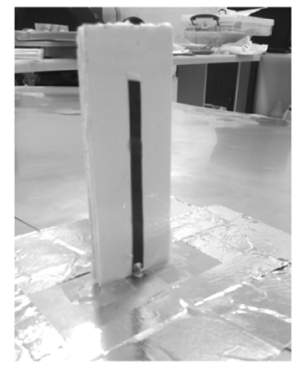

c)
Fig. 3. CNT buckypaper. (a) Prepared sample. (b) Irregularities of the sample toward the center. (c) CNT buckypaper monopole over the ground plane.

$S=1.6 \times 10^{-2} \mathrm{mg} / \mathrm{mm}^{2}$, the thickness results to be about $32 \mu \mathrm{m}$.

The sonication and the evaporation/filtration procedures are extremely critical for optimizing the CNT buckypaper performance. Large CNT agglomerates in the CNT solution will dominate leading to a brittle film, as well as a nonuniform evaporation produces a nonuniform sheet with holes, and consequently nonisotropic conductive performances. Fig. 3 shows the result of the process: the CNT buckypaper appears macroscopically uniform (a), but some undesired inconsistencies are present at the center of the sample (b) leading to poor and not uniform conductivity and mechanical properties.

\section{A. RF Characterization}

The dielectric properties of the CNT are strictly dependent on their deposition techniques. Concentration, orientation, number of layers, and material type are important parameters that affect the performances of the nanotubes, especially at RF. Although several characterization data have been presented, especially in dc, the dielectric properties at RF are not univocally defined, making it necessary to preliminarily characterize the produced buckypaper. Here, an indirect method, using both measurements and computer simulations, has been used to refine the different values available in the literature according to our specific sample. Based on the assumption that the CNT can be considered a lossy metal (with finite conductivity $\sigma$ ), several strips of the CNT buckypaper of size $80 \mathrm{~mm} \times 5 \mathrm{~mm}$ were cut out of the membrane and plated on foam substrates in order to form monopole antennas in the RFID UHF frequency band. Fig. 3(c) shows a photograph of one of the monopole antennas used in the measurement setup. A silver epoxy mixture was applied at the interface CNT antenna-SMA connector and cured to improve their electrical and mechanical connectivity. The input impedance of the CNT antennas has been measured by means of a vector network analyzer (VNA) (Rohde\&Schwarz ZVA8). In parallel, finite-difference time-domain (FDTD) simulations 

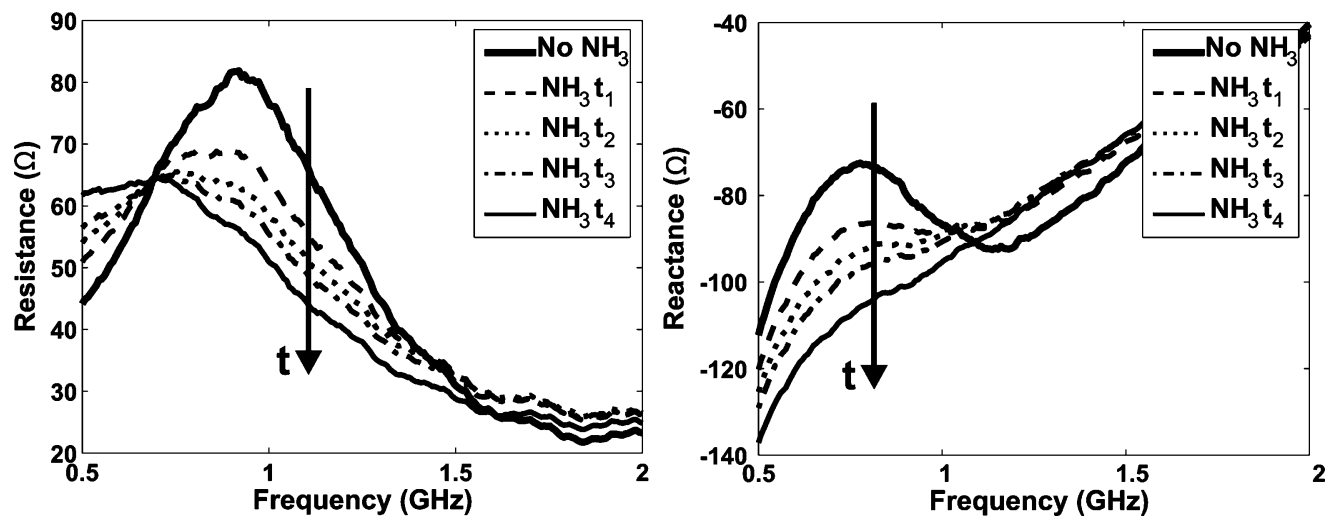

Fig. 4. $Z_{\text {in }}$ results of the monopole CNT antenna of Fig. 3 with and without the presence of the $\mathrm{NH}_{3}$. Here $t_{1}=1 \mathrm{~min}, t_{2}=5 \mathrm{~min}, t_{3}=10 \mathrm{~min}$, and $t_{4}=15 \mathrm{~min}$.
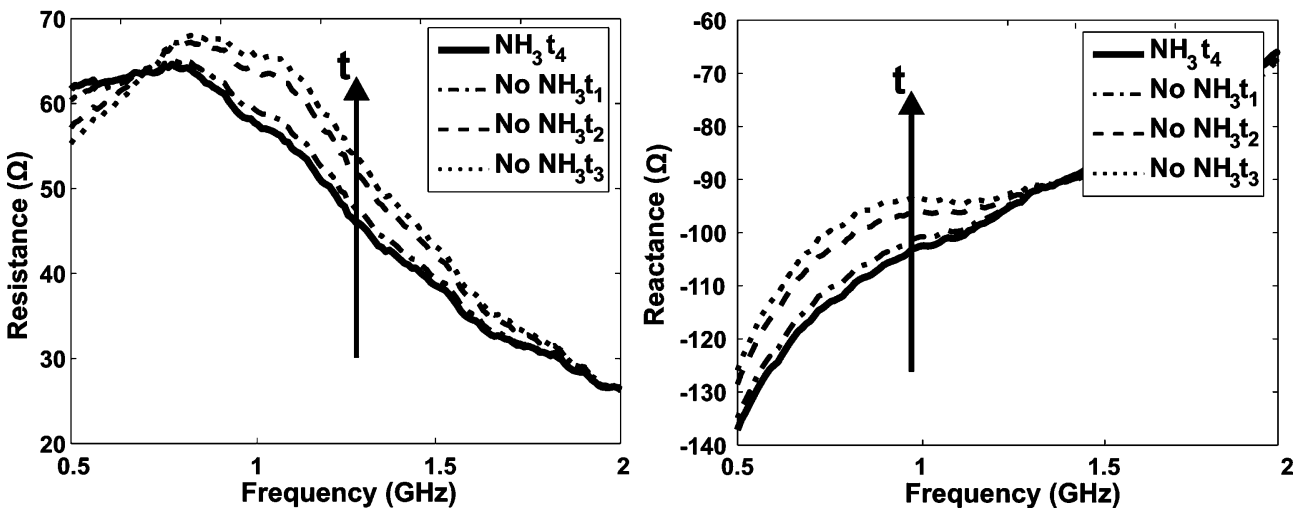

Fig. 5. $Z_{\text {in }}$ recovery features of the monopole CNT antenna of Fig. 3 after ammonia removal. Here $t_{1}=10 \mathrm{~min}, t_{2}=30 \mathrm{~min}$, and $t_{3}=60 \mathrm{~min}$.

were performed by varying the unknown $\sigma$ around the value available in literature. The CNT layer was accounted by a surface impedance model. A root mean square (rms) minimization has been finally applied in order to fit the measured and simulated input impedances and thus evaluate the conductivity. The optimum value obtained for the buckypaper after averaging on different monopole geometries was $\sigma \approx 2.5 \mathrm{~S} / \mathrm{m}$.

\section{B. Sensing Characterization}

The response of the CNT to the ammonia is investigated here by analyzing the variation of the radiation performances of one of the previous monopoles (size $80 \mathrm{~mm} \times 5 \mathrm{~mm}$ ) in term of both input impedance and transmission characteristics.

1) Input Impedance: Fig. 4 (thick line) shows the measured $Z_{\text {in }}$ of the CNT antenna before the exposure to ammonia. The monopole is strongly capacitive with a $-110 \leq j X_{\text {in }} \leq-70 \Omega$ and with a resistance $30 \leq R_{\text {in }} \leq 80 \Omega$.

A volume of $6 \mathrm{~mL}$ of commercial off-the-shelf $10 \%$ ammonia hydroxide was then guided into the $460-\mathrm{cm}^{3}$ plastic chamber surrounding the monopole. Upon the addition of the ammonia, a quick and strong variation of the monopole impedance was recorded. This is shown in Fig. 4, where $t_{1}$ implies the time the measurements were recorded right after the introduction of the gas. The effect is monotonic for both $R_{\text {in }}$ and $X_{\text {in }}$ during the whole exposure $t_{4}-t_{1} \approx 15 \mathrm{~min}$. As time progresses, slight saturation in the recorded measurements is observed.
The recovery of $Z_{\text {in }}$ to its initial value was monitored after removing the ammonia from the chamber (Fig. 5). As in the exposure process, the variation is monotonic, but much slower (here, $t_{3}-t_{1} \approx 1 \mathrm{~h}$ ). A slight difference between the input impedances before the exposure to ammonia and after the recovery phase is observed as well, demonstrating the quite irreversible effect of $\mathrm{NH}_{3}$ on the CNT. This effect is well known in the literature [15], and it is caused by the nature of gas absorption and desorption process to the nanotubes.

2) Transmission: Two identical CNT monopoles fabricated as before are now faced and separated by $20 \mathrm{~cm}$ to measure the transmission characteristics or $S_{21}$ with and without $\mathrm{NH}_{3}$. This measurement gives an evaluation of the variation of the gain in the presence of gas, which is proportional to $\left|S_{12}\right|^{2}$ between the two antennas [23]. Only one monopole is exposed to the ammonia, according to the setup previously described. The measured $S_{21}$ are shown in Fig. 6 upon adding $\mathrm{NH}_{3}$ (a) and upon its removal (b). The measurement times are defined similarly to those of Section II-B.1 with $t_{4}-t_{1} \approx 15 \mathrm{~min}$ in the exposure process and $t_{3}-t_{1} \approx 1 \mathrm{~h}$ for the recovery.

Without ammonia (Fig. 6 thick line), $S_{12}$ has an average value of about $\sim-40 \mathrm{~dB}$ in the UHF band, suggesting the low conductivity of the radiating structures. Similarly to the input impedance, $S_{12}$ changes monotonically in the presence of gas as time progresses. It is observed that a quick response takes place upon the addition of $\mathrm{NH}_{3}$, as well as saturation phenomena as $t \rightarrow t_{4}$. The transmission performance deteriorates as the 

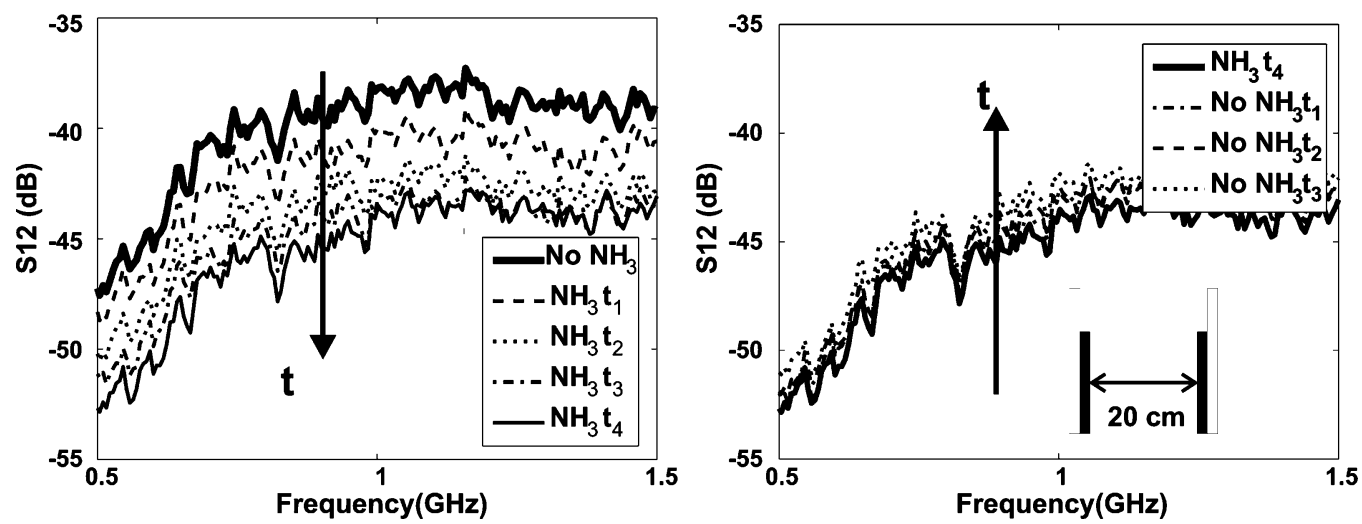

Fig. 6. $S_{12}$ variation between two identical CNT monopole antennas with and without the presence of the $\mathrm{NH}_{3}$.

antenna is exposed to $\mathrm{NH}_{3}$, losing almost $7 \mathrm{~dB}$. This effect seems to confirm a decrease in conductivity, as experienced in [10] and [15]. This can be explained considering that this gas is a reducing agent that injects electrons to the nanotubes (p-type) reducing the number of holes, and hence, the conductivity.

Recovery features are still present, but slower than in the previous experiment. The permanent effects of the gas are clearly visible: the recovery variation is about $4 \mathrm{~dB}$, almost half of the one achieved during the exposure process.

From these experiments, it is hence clear that the presence of ammonia affects both the impedance and gain of the monopole, potentially promising interesting applications in the RFID domain.

\section{RFID-SENSING EQUATIONS}

It is assumed to denote with $\Psi(t)$ the concentration of ammonia in the air that has to be detected by the RFID platform.

The well-known [24] two-way reader-tag link may be rewritten, making explicit the dependence on the variation of local parameters with the purpose to obtain some sensing indicators, which can be directly derived by the reader's measurements [11]. The power collected at the microchip (1) and the power backscattered by the tag toward the reader (2) and collected by it are

$$
\begin{aligned}
& P_{R \rightarrow T}[\Psi]=\left(\frac{\lambda_{0}}{4 \pi r}\right)^{2} P_{\mathrm{in}} G_{R}(\theta, \phi) G_{T}[\theta, \phi, \Psi(t)] \tau\left([\Psi(t)] \eta_{p}\right. \\
& P_{R \leftarrow T}[\Psi]=\frac{1}{4 \pi}\left(\frac{\lambda_{0}}{4 \pi r^{2}}\right)^{2} P_{\mathrm{in}} G_{R}^{2}(\theta, \phi) \eta_{p}^{2} r c s_{T}[\theta, \phi, \Psi(t)]
\end{aligned}
$$

where $r$ is the reader-tag distance, $G_{R}(\theta, \phi)$ is the gain of the reader antenna, and $G_{T}[\theta, \phi, \Psi(t)]$ is the gain of the tag's antenna integrating the nanotube film. $P_{\text {in }}$ is the power entering the reader's antenna, $\eta_{p}$ is the polarization mismatch between the reader and the tag, and $\tau[\Psi(t)]$ is the power transmission coefficient of the tag

$$
\tau[\Psi]=\frac{4 R_{\text {chip }} R_{A}[\Psi]}{\left|Z_{\text {chip }}+Z_{A}[\Psi]\right|^{2}}
$$

with $Z_{\text {chip }}$ input impedance of the RFID integrated circuit (IC) and $Z_{A}$ input impedance of the antenna. $r c s_{T}$ is the tag's radar cross section, related to the modulation impedance $Z_{\bmod }$ of the microchip to encode the low and high digital state

$$
\operatorname{rcs}_{T}[\Psi]=\frac{\lambda_{0}^{2}}{4 \pi} G_{T}^{2}[\theta, \phi, \Psi(t)]\left(\frac{2 R_{A}[\Psi]}{\left|Z_{\bmod }+Z_{A}[\Psi]\right|}\right)^{2} .
$$

The backscattered power $P_{R \leftarrow T}$ is directly measurable by the reader in terms of the received signal strength indicator (RSSI), here assumed to correspond [24], [25] to the binary modulating state having the highest $r c s_{T}\left(Z_{\text {mod }}=Z_{\text {chip }}\right)$.

A first parameter that can be measured by the reader is the turn-on power $P^{\mathrm{to}}[\Psi]$, e.g., the minimum input power $P_{\text {in }}$ through the reader's antenna forcing the tag to respond. It can be derived from (1) as follows, considering $P_{R \rightarrow T}=P_{\text {chip }}$ that is the microchip sensitivity:

$$
P^{\mathrm{to}}[\Psi]=\left(\frac{\lambda_{0}}{4 \pi d}\right)^{-2} \frac{P_{\text {chip }}}{G_{R}(\theta, \phi) \eta_{p} G_{T}[\theta, \phi, \Psi] \tau[\Psi]} .
$$

From turn-on measurement it is possible to extract the realized gain of the tag $G_{\tau}[\Psi]$, e.g., the gain of the tag scaled by the mismatch to the IC, strictly correlated to the physical variation of the local environment

$$
G_{\tau}[\Psi]=\left(\frac{4 \pi d}{\lambda_{o}}\right) \frac{P_{\text {chip }}}{G_{R}\left(\theta_{R}, \phi_{R}\right) P^{\text {to }}} .
$$

Finally, forward (1) and backward (2) powers may be combined at turn-on with the purpose to drop out the influence of the distance and of the reader and tag gains and orientation [26], [27]. A nondimensional indicator, denoted as an analog identifier (AID), can hence be introduced

$$
\operatorname{AID}[\Psi]=\frac{P_{\text {chip }}}{2 \sqrt{P_{R \leftarrow T}[\Psi] P^{\mathrm{to}}[\Psi]}}=R_{\text {chip }}\left|Z_{\text {chip }}+Z_{A}[\Psi]\right|^{-1}
$$

and it is very useful when the interrogation setup changes (position and orientation) in successive measurements. The AID, in fact, just depends on the impedance mismatch and is immune to the interrogation modalities. 


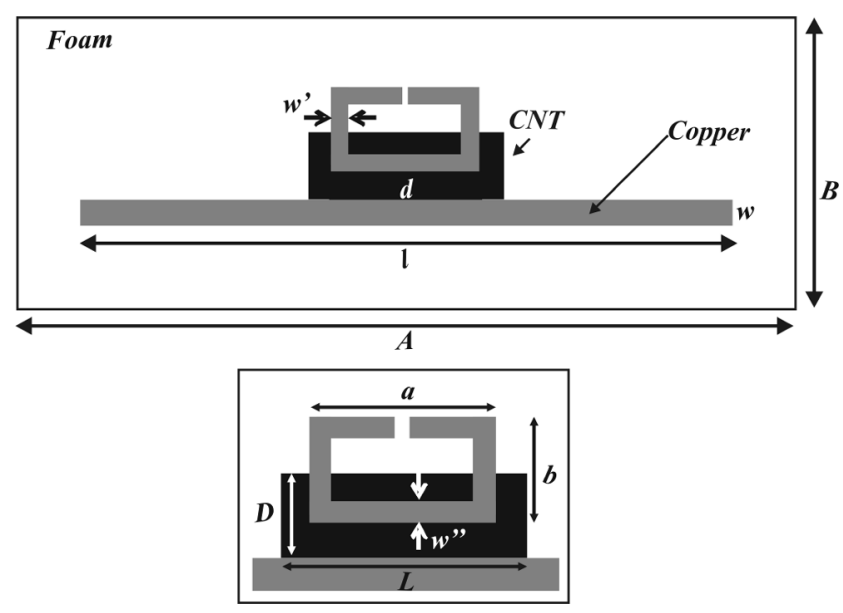

Fig. 7. RFID tag: inductively coupled loop with a rectangular CNT load. The matching to the IC can be achieved for example by acting on the parameter $d$ and $a$ and by fixing the others.

The two indicators in (5) and (7) may be used as data inversion curves $p[\Psi(t)]$ between the measured data and the evolution $\Psi(t)$ of the process. They are independent since the tag undergoes change in gain and impedance mismatch.

\section{RFID GAS SENSOR: DESIGN AND PROTOTYPE}

There are several options to achieve a gas-sensitive tag taking advantage of the sensing feature of the CNT demonstrated in Section II. A first classification could be done considering the CNT as a part of the antenna radiating structure, e.g., an antenna totally or partially made of nanotubes, or considering instead the buckypaper as a doping component of the tag, e.g., a variable load properly placed in close proximity of the radiating elements [10]. Both solutions offer advantages and drawbacks; the best tradeoff between communication and sensing must be finally adopted. By considering the CNT as a radiating element, it is possible to maximize the gas sensitivity of the device; however, lowering the antenna efficiency and the activation distance of the RFID tag. These two parameters, together with the cost, are essential in RFID systems. The other solution, on the contrary, offers better communication and cost performances, but could provide a weaker sensitivity to the gas.

In this work, CNT film is used as a loading impedance of a conventional RFID passive tag [10]. The considered antenna layout is a copper dipole with an inductively coupled loop [28], matched to an NXP IC with complex impedance $Z_{\text {chip }}=16-$ $j 148 \Omega$ and sensitivity $P_{\text {chip }}=-15 \mathrm{dBm}$. In this geometry (Fig. 7), the strength of the coupling is controlled by the distance $d$ between the loop and the radiating body, as well as by the shape factor $(a, b)$ of the loop. A rectangular sample of CNT buckypaper (of size $D \times L$ ) is thus placed in the space between the loop and the radiating dipole, this area being the most critical for the antenna's radiation performances.

The CNT film affects the coupling between the two structures, modifying the current's distribution, and consequently the input impedance of the tag. More specifically, the presence of the CNT film between the dipole and the loop produces a cur-

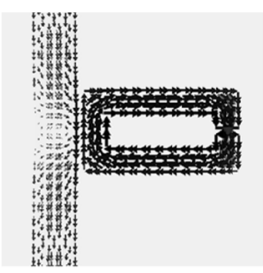

(a)

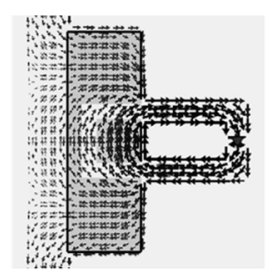

(b)

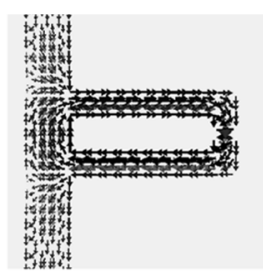

(c)
Fig. 8. Current distribution simulated by FDTD method. (left) Standard loop fed dipole (no CNT), (center) loop fed dipole integrating CNT, (right) conventional T-match dipole. Although the magnitude is quite similar, the currents are sensibly different in the CNT area, making the antenna very similar to the T-matched one.

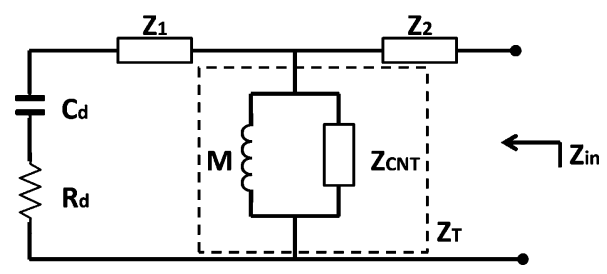

Fig. 9. RFID radio-sensor integrating CNT equivalent circuit. A T-model has been adopted to represent the transformer in [28]. The parameters $R_{d}, C_{d}$, and $L_{d}$ (for clarity, grouped in $Z_{1}$ ) give the input impedance $Z_{A}$ of the radiating body near its (series) resonance.

rent flow between the two copper structures (Fig. 8), whose effect competes against the one produced by the inductive coupling: for extremely low conductivity, the prevailing effect is inductive, while, as well as the conductivity increases, the ohmic effect becomes stronger and the structure resembles, from a current point of view, a T-match feed [28]. Two different effects are hence present: ohmic currents that flow along $d$ and a variation of the shape factor of the loop (see Fig. 8, center). As visible, the currents tend to reduce the length of the loop, flowing all along the surface and the perimeter of the CNT area. Both real and imaginary parts of the input impedance are accordingly dependent on the lossy CNT material.

\section{A. Circuit Model}

Under the hypothesis that the CNT can be considered as a lossy metal with variable conductivity, the input impedance of the radio sensor and its variation with the CNT conductivity can be described by the general equivalent circuit in Fig. 9. The circuit has been obtained through a proper modification of the topology described in [28] whose impedance transformer is replaced here by its $\mathrm{T}$-model equivalent by introducing the series impedances

$$
Z_{1}=j \omega L_{d}-Z_{T}
$$

and

$$
Z_{2}=j \omega L_{l}-Z_{T}
$$

where $Z_{T}=j \omega M \| Z_{\mathrm{CNT}}$ is the modified impedance of transformation, which takes into account the presence of CNT $\left(Z_{\mathrm{CNT}}\right)$ and of the mutual inductance $M$ only related to the geometry of the structures [30]. $L_{l}$ is the loop inductance, and finally $Z_{A}=R_{d}+1 / j \omega C_{d}+j \omega L_{d}$ the input impedance of the 
radiating body around its first resonance. The presence of the CNT film impacts on the coupling between the dipole and the loop so that the input impedance at the chip port is finally given by

$$
Z_{\text {in }}=j \omega L_{l}+\frac{\left(Z_{T}\right)^{2}}{Z_{A}} .
$$

$Z_{\mathrm{CNT}}$ is related to various CNT's parameters, such as its size, its conductivity, and the consequent skin effect $\delta_{s}$. It is roughly proportional to the surface impedance of imperfect conductors [31] and can be evaluated by means of best fitting of the numerically computed input impedance to the circuital expression in (8) in order to take into account the specific shape factor of the film. For the particular structure described in Fig. 7,

$$
Z_{\mathrm{CNT}}=f\left(\sigma, \text { shape } \text { factor }_{\mathrm{CNT}}\right) \approx K_{1} \frac{(1+j)}{\sigma \delta_{s}}
$$

where $\delta_{s}=\sqrt{2 /\left(\omega \mu_{r} \sigma\right)}$ is the skin depth, $\mu_{r}=1$ is the relative magnetic permeability of conductor and $\sigma$ its conductivity. The loop inductance $L_{l}$ is mainly related to the geometric features of the structures [30]; however, as shown in Fig. 8 (center), the presence on the CNT film in the inner part of the loop strongly modifies its current distribution, essentially reducing the overall perimeter of the effective current loop. The width of the horizontal segment $w^{\prime \prime}(\sigma)$ (in Fig. 7) can thus be properly corrected to include this effect as

$$
w^{\prime \prime} \approx w^{\prime}+\left(K_{2} \sigma+K_{3}\right) \cdot a
$$

with weights $K_{2,3}$ to be defined through linear fitting.

It is worth noticing that for extremely low conductivity $(\sigma \rightarrow$ $0)$, the CNT impedance becomes an open circuit $\left(Z_{\mathrm{CNT}} \rightarrow \infty\right)$ and $Z_{T} \rightarrow M$ as for the standard inductively coupled structure, while for $\sigma \rightarrow \infty, Z_{\mathrm{CNT}} \rightarrow 0$ and $Z_{T} \rightarrow 0$ (ohmic contact), giving rise to a $\mathrm{T}$-match feed.

The circuit model in Fig. 9 is of general application and could be used to support the design of other gas sensors integrating CNT with different conductivity $\sigma$, as well as to understand how this last parameter affects the tag's radiation performances. It is, however, worth noticing that since the circuit represents a hybrid between the T-match and the inductively coupled loop, it is reasonably valid only for relative low values of $\sigma$ : as the conductivity increases, a more suitable equivalent circuit model should be used to describe the T-match like structure [28].

\section{B. Parametric Analysis}

Fig. 10 shows an example of parametric analysis, computed by the equivalent circuit model, for the loop-driven dipole in Fig. 7 loaded by a $30 \mathrm{~mm} \times 10 \mathrm{~mm}$ CNT buckypaper with $\sigma=$ $2.5 \mathrm{~S} / \mathrm{m}$, as evaluated in Section II, when varying the shape factor of the loop $a$ and its distance from the dipole $d$. Such analysis has been compared with the one produced by the fullwave FDTD simulation. For the particular geometry of the CNT, the following weights have been found by linear fitting $K_{1}=$ $1.7, K_{2}=6 \cdot 10^{-2}$, and $K_{3}=-4 \cdot 10^{-2}$.
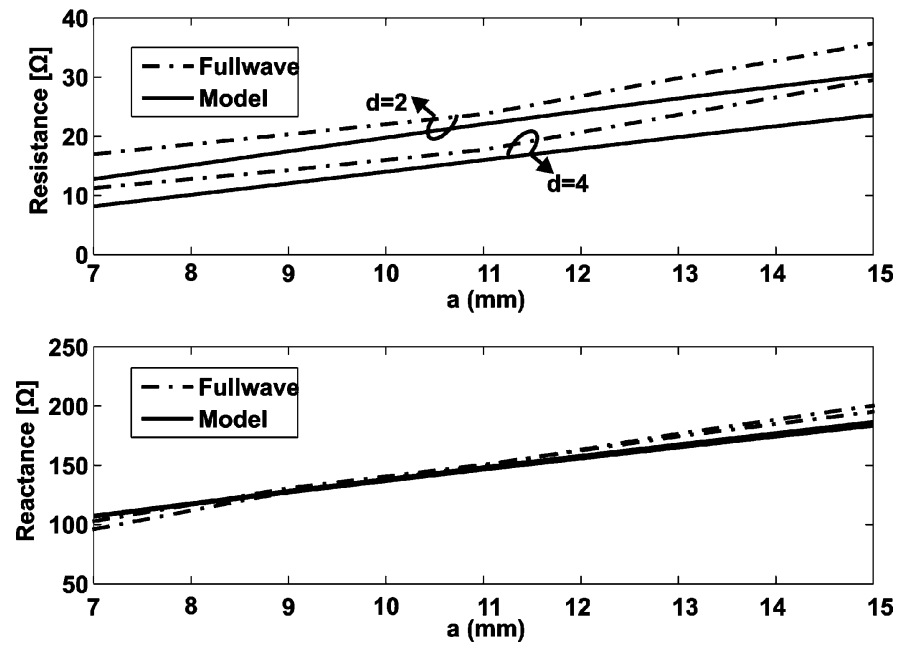

Fig. 10. Parametric analysis of the input impedance varying the shape factor of the loop and its distance from the vertical dipole, having fixed (size in millimeters) $l=160, w=5, L=30, w^{\prime}=2, b=22$, and $D=10$.
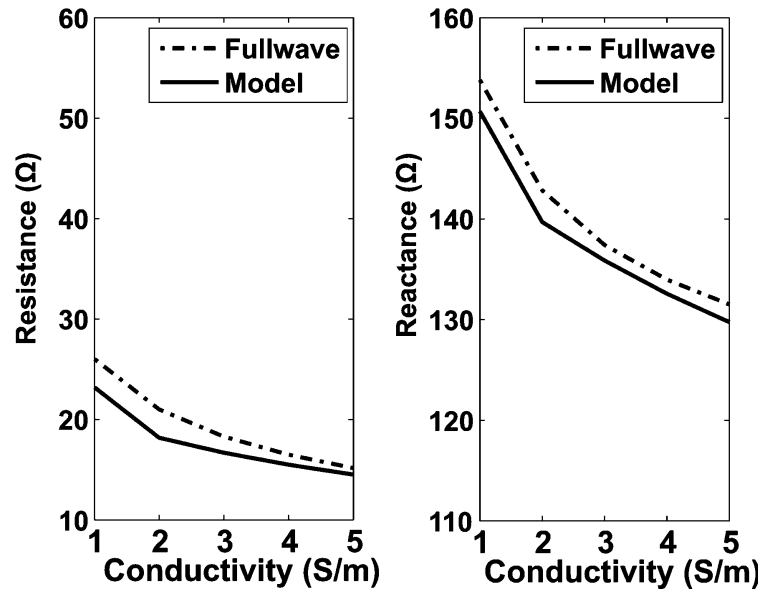

Fig. 11. Change of the input impedance of the loaded RFID tag with conductivity.

The input reactance is nearly unaffected by the loop-dipole distance $d$. For a fixed loop size, instead, the resistance reduces when the loop-dipole distance increases. For the particular choice of parameters as $a=10 \mathrm{~mm}$ and $d=2 \mathrm{~mm}$, the predicted antenna impedance is $Z_{\text {in }}(a, d)=17+j 140[\Omega]$, very close to the required value to match the NXP IC.

For this size of the loop, the variation of the input impedance at $915 \mathrm{MHz}$ with conductivity is shown in Fig. 11 for $\sigma=$ $[1,5] \mathrm{S} / \mathrm{m}$.

As expected from physical considerations, the variation of CNT conductivity impacts both the real and imaginary parts of the input impedance. They monotonically decrease as the conductivity increases according to different variation profiles. In percentage terms, the effect is stronger on the input resistance since the reactance is mainly governed by the shape factor of the loop, as indicated in Fig. 8. Moreover, the presence of the CNT will modify the losses of the tag, also producing a variation of gain. 


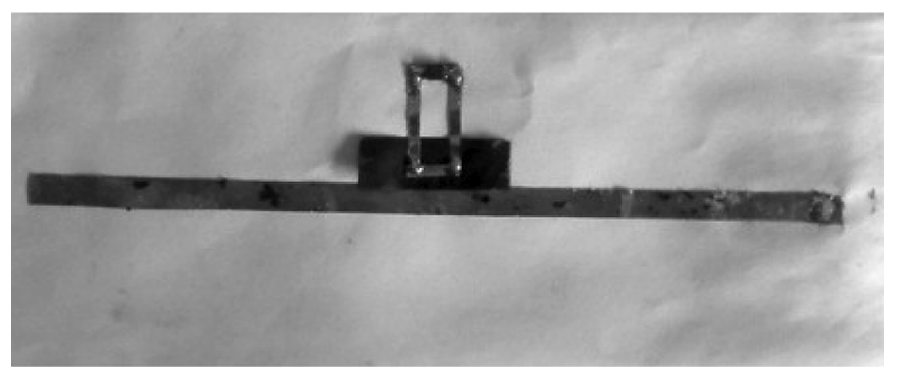

Fig. 12. Photograph of prototype. Size in millimeters: $A=180, B=80$, $l=160, w=5, L=30, w^{\prime}=2, a=10, b=22, D=10$, and $d=2$.
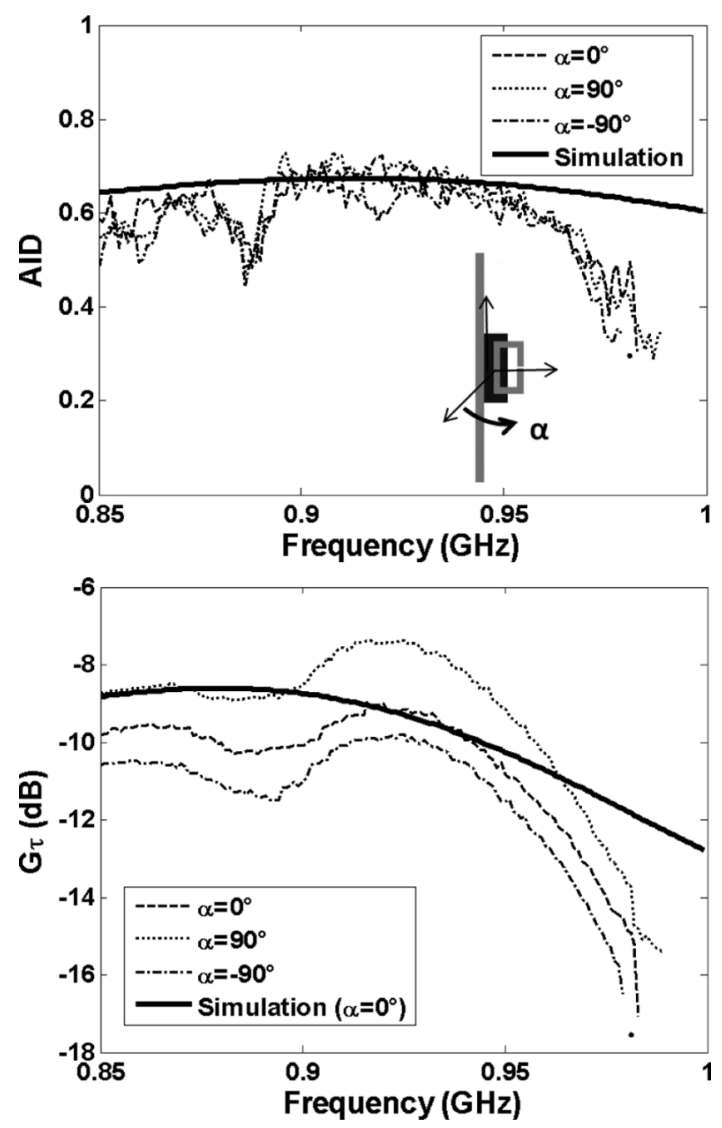

Fig. 13. Simulation and measurement results for AID and $G_{\tau}$ for the CNTloaded RFID prototype.

\section{Prototype}

A first prototype of the tag is shown in Fig. 12. Voyantic Tagformance is used for the characterization of the RFID response of the tag. The interrogator antenna is a Kushcraft patch having a 6-dBi gain and a circular polarization. The reader's antenna-tag distance is fixed to $r=63.5 \mathrm{~cm}$. Both the reader and the tag are $101 \mathrm{~cm}$ high from the floor. All the measurements have been performed in an anechoic chamber.

The measured AID (7) and realized gain $G_{\tau}(6)$, as defined in Section III, are shown in Fig. 13 for three different orientations. The AID is independent of the reader-tag mutual position [26], while the realized gain is rather sensitive to the reader-tag orientation due to the variable gain of the tag over the $H$-plane. Simulation and measurements have been compared along the

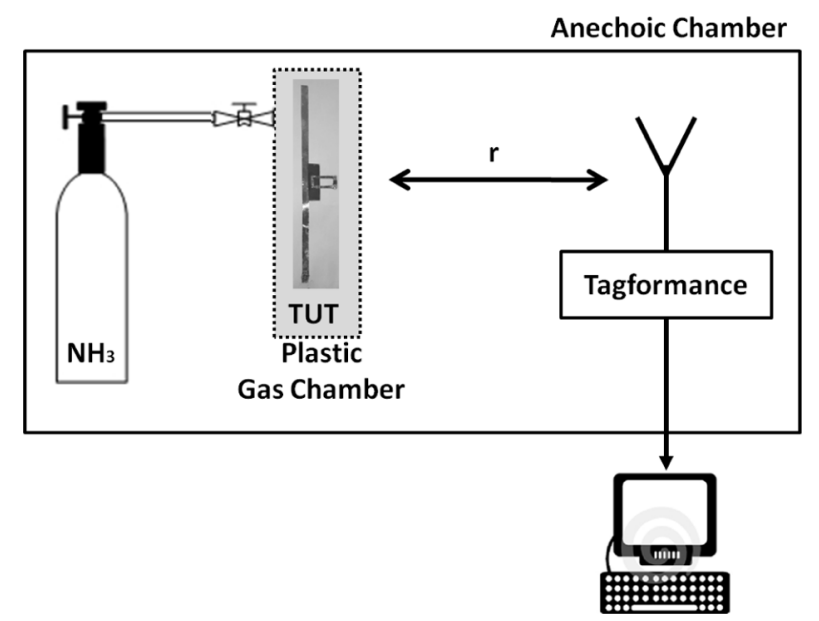

Fig. 14. Measurement setup comprising the remotely controlled reader, the ammonia tank connected to the gas chamber, and the RFID TUT. The reader's antenna-tag distance is fixed to $r=63.5 \mathrm{~cm}$.

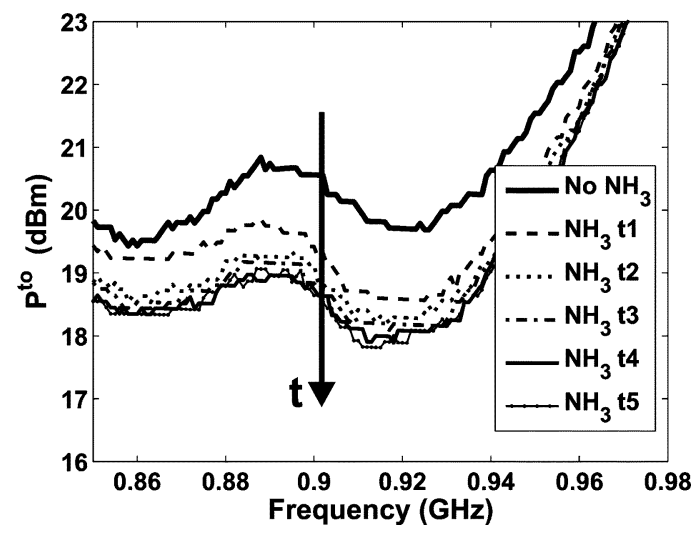

Fig. 15. Turn-on power of the CNT loaded RFID tag with $\mathrm{NH}_{3}$.

TABLE I

EXPOSURE AND RECOVERY MEASUREMENT TIME-STEPS

\begin{tabular}{c|c}
\hline Exposure time & Recovery time \\
\hline$t_{1}=1 \mathrm{~min}$ & $t_{1}=5 \mathrm{~min}$ \\
$t_{2}=4 \mathrm{~min}$ & $t_{2}=13 \mathrm{~min}$ \\
$t_{3}=6 \mathrm{~min}$ & $t_{3}=40 \mathrm{~min}$ \\
$t_{4}=9 \mathrm{~min}$ & $t_{4}=50 \mathrm{~min}$ \\
$t_{5}=15 \mathrm{~min}$ & $t_{5}=60 \mathrm{~min}$ \\
\hline
\end{tabular}

normal direction, e.g., when the tag is placed exactly in front of the reader. Although there are some deviations at the lower and higher frequencies, basically due to the nonlinearity of the IC, they reasonably fit around the designed frequency $915 \mathrm{MHz}$, as shown in Fig. 13.

It is important to point out the effect of the CNT buckypaper on the radiation performances of the tag. The maximum measured realized gain is around $-7 \mathrm{~dB}$, which implies a simulation gain of about $-6 \mathrm{~dB}$, worse than typical gain for this kind of antenna $(0-2 \mathrm{~dB})$ due to the presence of a lossy loading.

The maximum reading distance $r_{\text {Max }}$ can be evaluated from (1) enforcing $P_{R \rightarrow T}=P_{\text {chip }}$. Taking into account the measurement results and considering a reader emitting up to $4-\mathrm{W}$ effec- 


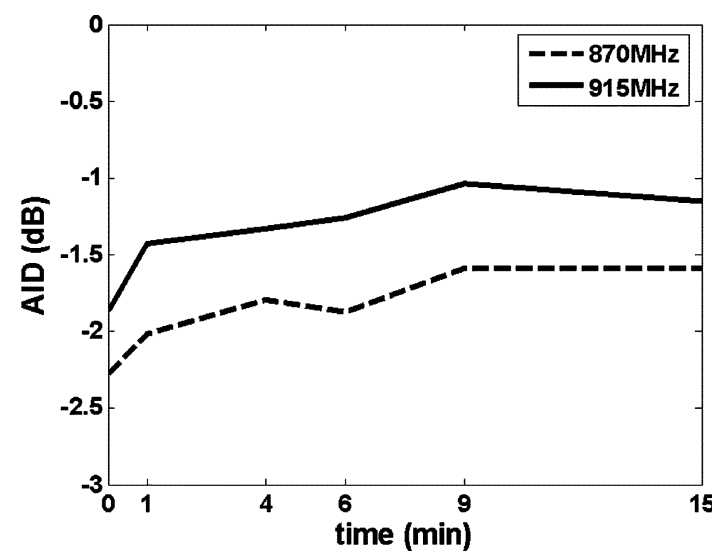

Fig. 16. $G_{\tau}$ and AID for the CNT-loaded RFID prototype with $\mathrm{NH}_{3}$.

tive isotropic radiated power (EIRP) in linear polarization, the estimated maximum read distance is $4 \mathrm{~m}$.

\section{SENSING CHARACTERIZATION}

To test the sensing performances of the tag, it has been placed into a $684-\mathrm{cm}^{3}$ plastic gas chamber, and a volume of $6 \mathrm{~mL}$ of $10 \%$ ammonia hydroxide was guided into it (Fig. 14). The reference condition for the tag, without ammonia, accounts for the presence of the gas chamber in close proximity of the radiating structures.

Fig. 15 shows the results of the $P^{\text {to }}[\Psi]$ for the tag-under-test (TUT) following the exposure to $\mathrm{NH}_{3}$. The measurement timesteps are indicated in Table I. An immediate and sharp variation of response is observed at $t_{1}=1 \mathrm{~min}$ followed by a saturation. Within the band of interest, the behavior is monotonic, with about $2 \mathrm{~dB}$ of overall variation. Since the turn-on power is inversely proportional to the realized gain $G_{\tau}=G_{T} \cdot \tau$ of the tag, the diagrams in Fig. 15 suggest that the tag performances improve along with the exposure to ammonia. This behavior seems to be in contrast with the two CNT-monopoles experiment in Fig. 6, wherein $|S 12|^{2}$ reduces as $\left[\mathrm{HN}_{3}\right]$ increases. A possible explanation is that the presence of ammonia tends to improve the impedance matching $(\tau)$ between the tag and the microchip. A more detailed chemical analysis of the absorption should be nevertheless required to fully understand this effect.

It is worth noticing that the time required to take a complete measurement of data $(840-980 \mathrm{MHz})$ is approximately $90 \mathrm{~s}$, and thus it may be comparable with the response time of the sensor (approximately 2 min according to [15]). This could affect the accuracy of the data especially at higher frequencies since the absorbtion of the ammonia, and thus the electromagnetic features of the CNT, are changing in the meanwhile the measurement is performed. However, for the two UHF RFID frequencies considered in this paper (European and American bands), the measurement time can be assumed to be less than $40 \mathrm{~s}$, and hence, the measurement artifact could be considered negligible.

The visible variation of $G_{\tau}[\Psi]$ and $\operatorname{AID}[\Psi]$ in the presence of $\mathrm{NH}_{3}$ (Fig. 16) confirms that both input impedance and gain are affected by the gas. As expected, the latter indicator is more sensitive to $\mathrm{NH}_{3}$ than AID due to the additional dependence on the tag's gain $G_{T}[\theta, \phi, \Psi]$.
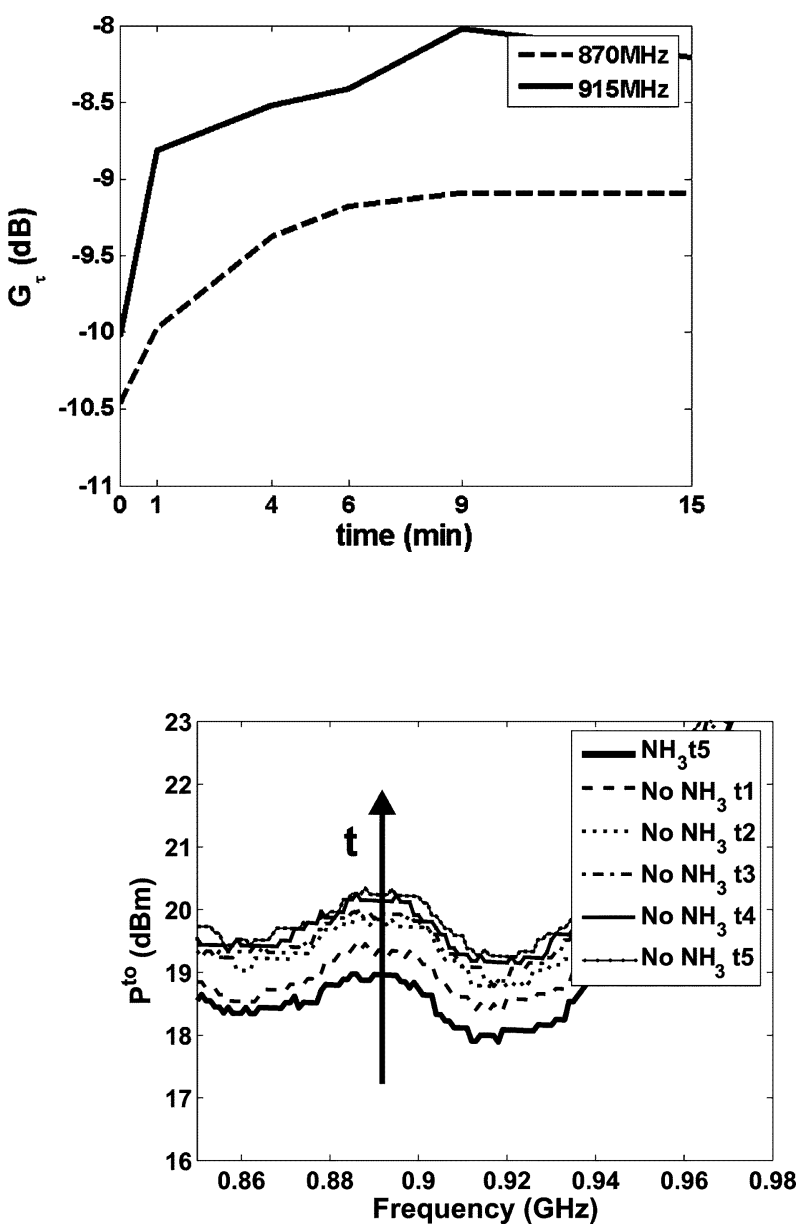

Fig. 17. Turn-on power of the CNT loaded RFID tag—recovery.

It is useful to introduce the percentage variation of realized gain $\Delta G_{\tau}$ and AID $\Delta$ AID referred to the initial state $t_{0}$ such as without ammonia in the air

$$
\Delta \xi\left(t_{i}\right)=\frac{\xi\left(t_{i}\right)-\xi\left(t_{0}\right)}{\xi\left(t_{0}\right)} \cdot 100
$$

with $\xi=\left\{G_{\tau}, \mathrm{ADD}\right\}$.

The maximum variation for both indicators arises just after the ammonia introduction, more specifically, $\Delta G_{\tau}\left(t_{1}\right)=30 \%$ and $\Delta \operatorname{AID}\left(t_{1}\right)=10 \%$. The total variation is $\Delta G_{\tau}\left(t_{5}\right)=60 \%$ and $\Delta \mathrm{AID}\left(t_{5}\right)=20 \%$ and seems to be maximum at $915 \mathrm{MHz}$.

\section{A. Recovery Features}

Figs. 17 and 18 show the recovery plots of $P^{\mathrm{to}}[\Psi], G_{\tau}[\Psi]$, and $\mathrm{ADD}[\Psi]$ versus frequency after having removed the ammonia. A monotonic recovery characteristic is present with a slower response (see Table I for all the recovery time steps), mainly occurring in the first $15 \mathrm{~min}$.

Although $G_{\tau}$ shows clear recovery features, the AID seems quite insensitive to the phenomena, especially at $870 \mathrm{MHz}$. This issue could be related to the fact that the dependence of the latter indicator only on the input impedance of the tag makes it less sensitive to the physical variation of the antenna. 


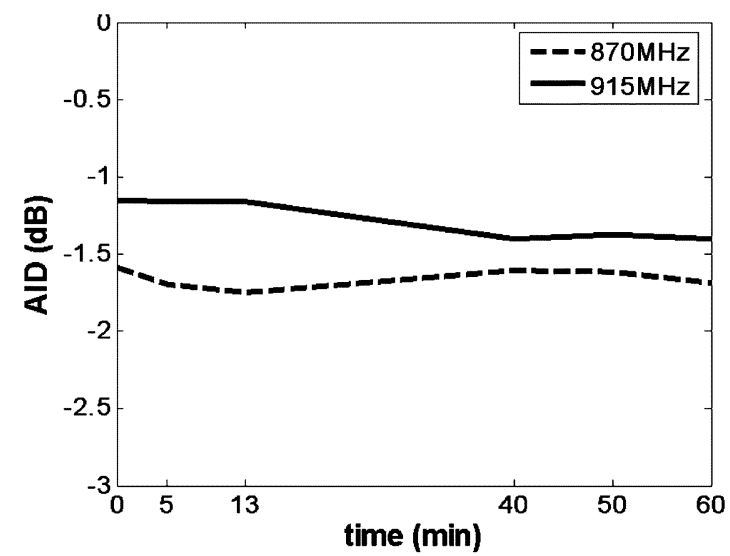

Fig. 18. $G_{\tau}$ and AID for the CNT-loaded RFID prototype with $\mathrm{NH}_{3}$-recovery.

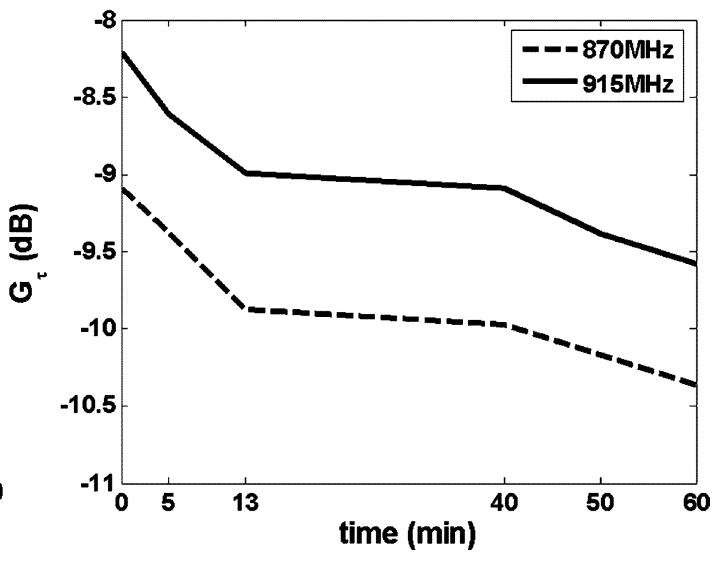

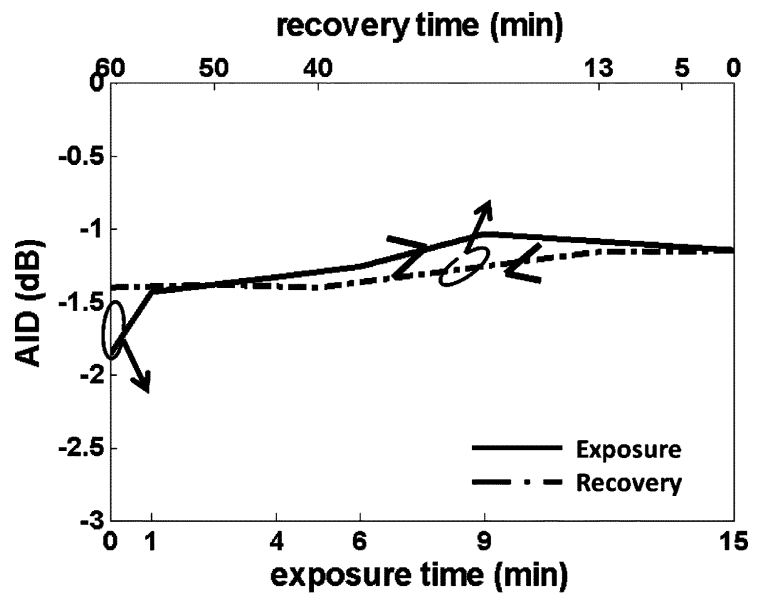

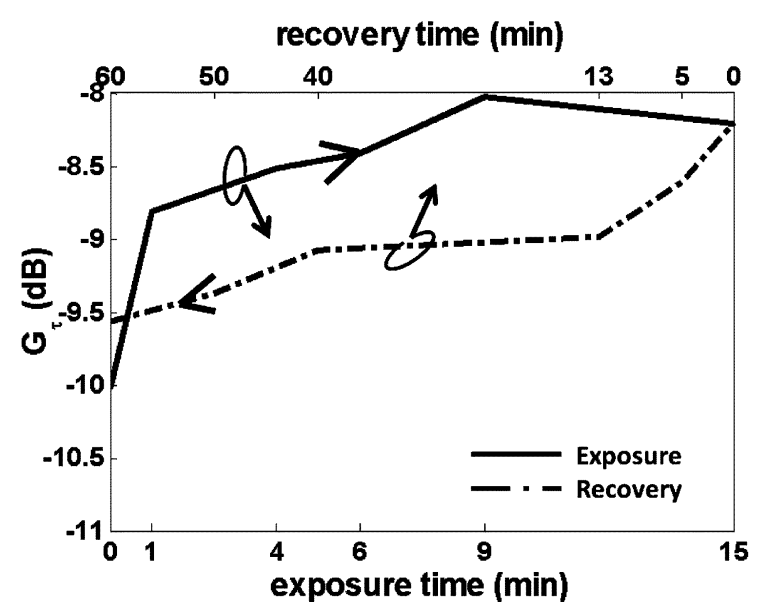

Fig. 19. $G_{\tau}$ and AID at $915 \mathrm{MHz}$ during the exposure and the recovery process.

When considering the whole cycle, it is apparent that the presence of ammonia irreversibly modifies the tag's radiation performances, producing an hysteresis in both input impedance and gain (Fig. 19). A difference of about $10 \%$ in the turn-on power is indeed observed between the initial condition (without ammonia) and the final condition (after recovery).

\section{Discussion AND CONCLUSIONS}

The complete integration of CNT film into an RFID tag has been here reported for the first time aiming at designing and testing a fully working passive gas radio sensor. Simplified fabrication procedures, as well as indirect RF characterizations for the CNT buckypaper, have been proposed with promising results in term of feasibility and accuracy. The proof of concept of using a CNT-antenna and CNT-based passive RFID tag as $\mathrm{NH}_{3}$ wireless sensors has been theoretically and experimentally verified in spite of the strikingly low $\sigma$ of the tested samples.

Beside the promising early results, the optimization of this class of chemical-doped RFID tags is still prone to considerable improvements concerning the technology process to produce the CNT buckypaper load, the antenna design, and not least, the interrogation algorithm.

The proposed fabrication procedure seems indeed to offer great opportunities in term of cost and simplicity, but it should be better controlled and optimized in order to get defect-free and reproducible sensitive films at the cost of the lowest CNT amount. Depending on the preparation technique and process, in fact, the properties and the behavior of the sensors can vary significantly, and this is a very crucial issue for the large-scale production of CNTs-based devices. Furthermore, in order to design efficient sensing systems, it is necessary to quantify, and especially to enhance, the gas sensitivity of the buckypaper, as well as its response time and selectivity. A functionalized CNT should be considered for this purpose. Possible improvements of the proposed evaporation technique can be achieved by producing a smaller area of buckypaper in order to better control the uniformity of the deposition and reduce the required amount of nanotubes. The long recovery time, which could be a limiting issue for real-time monitoring of oscillating events, could instead find interesting application for a one-shot sensor, e.g., by taking advantage of the permanent physical modifications especially visible in the AID response.

In order to fully control the tag's behavior in time-varying boundary conditions, the electromagnetic design of the radio sensor needs further refinements, for instance, by employing multiphysics models. Recent studies [4] demonstrate the possibility to control and enhance the sensitivity and the dynamic 
range of a passive RFID sensor just in the design phase by properly choosing the IC family and the process's realization, thus forcing the antenna to have the best impedance matching to the RFID IC.

Finally, the interrogation of the tag in real-world environments could present big challenges, especially concerning the natural fluctuation of the electromagnetic responses caused by the multipath. If the measurement is performed in static conditions, e.g., the mutual position between the reader and the sensor tag remains unchanged during the whole phenomenon to monitor, or similarly, a same measurement setup can be exactly replicated in successive readings, the turn-on indicator gives a feasible and sharp response. More in general, the AID metric could be a solution to reduce the reading uncertainties, but further effort is required to better enhance its dynamic range in a controlled way.

\section{ACKNOWLEDGMENT}

The authors would like to thank the Georgia Tech Nanotechnology Research Center, Atlanta, for offering help in the fabrication processes.

\section{REFERENCES}

[1] J. Dowling, M. M. Tentzeris, and N. Beckett, "RFID-enabled temperature sensing devices: A major step forward for energy efficiency in home and industrial applications?," in IEEE MTT-S Int. Microw. Workshop on Wireless Sensing, Local Positioning, and RFID, Sep. 2009, pp. $1-4$.

[2] S. Caizzone, C. Occhiuzzi, and G. Marrocco, "Multi-chip RFID antenna integrating shape-memory alloys for detection of thermal thresholds," IEEE Trans. Antennas Propag., to be published.

[3] V. Lakafosis, A. Rida, R. Vyas, L. Yang, S. Nikolaou, and M. M. Tentzeris, "Progress towards the first wireless sensor networks consisting of inkjet-printed, paper-based RFID-enabled sensor tags," Proc. IEEE, vol. 98, no. 9, pp. 1601-1609, Sep. 2010.

[4] C. Occhiuzzi, G. Contri, and G. Marrocco, "RFID STENTag for passive vascular monitoring," in Proc. 5th Eur. Antennas Propag. Conf., Apr. 11-15, 2011, pp. 3476-3478.

[5] R. A. Potyrailo, C. Surman, R. Chen, S. Go, K. Dovidenko, W. G. Morris, E. Holwitt, V. Sorola, and J. L. Kiel, "Label-free biosensing using passive radio-frequency identification (RFID) sensors," in SolidState Sens., Actuators, Microsyst. Int. Conf., Jun. 2009, pp. 2378-2380.

[6] Y. Ikemoto, S. Shingo, O. Hiroyuki, M. Hiroki, X. Lin, I. Hideo, and A. Hajime, "Force sensor system for structural health monitoring using passive RFID tags for structural health monitoring," in 2nd IEEE Int. Interdisciplinary Portable Inform. Devices Conf. and 7th IEEE Polymers and Adhesives in Microelectron. and Photon. Conf., Aug. 2008, pp. $1-6$.

[7] C. Occhiuzzi, C. Paggi, and G. Marrocco, "RFID tag antenna for passive strain sensing," in Proc. 5th Eur. Antennas Propag. Conf., Apr. 11-15, 2011, pp. 2306-2308.

[8] C. Occhiuzzi, S. Cippitelli, and G. Marrocco, "Modeling, design, and experimentation of wearable RFID sensor tag," IEEE Trans. Antennas Propag., vol. 58, no. 8, pp. 2490-2498, Aug. 2010.

[9] J. Siden, G. Jinlan, and B. Neubauer, "Microstrip antennas for remote moisture sensing using passive RFID," in Asia-Pacific Microw. Conf., Singapore, Dec. 2009, pp. 2375-2378.

[10] L. Yang, R. Zhang, D. Staiculescu, C. P. Wong, and M. M. Tentzeris, "A novel conformal RFID-enabled module utilizing inkjet-printed antennas and carbon nanotubes for gas-detection applications," IEEE Antennas Propag. Lett., vol. 8, pp. 653-656, 2009.

[11] G. Marrocco, "Pervasive electromagnetics: Sensing paradigms by passive RFID technology," IEEE Wireless Commun., vol. 17, no. 6, pp. 10-17, Dec. 2010.
[12] M. I. Baraton, Sensors for Environment, Health and Security. Berlin, Germany: Springer, 2009, vol. XXIV, pp. 500-500.

[13] P. Avouris, J. Appenzeller, R. Martel, and S. J. Wind, "Carbon nanotube electronics," Proc. IEEE, vol. 91, no. 9, pp. 1784-12772, Nov. 2003.

[14] Y. Wang and J. T. W. Yeow, "A review of carbon nanotubes-based gas sensors," J. Sens., vol. 2009, 24 pp, Art. ID 493904.

[15] K. G. Ong, K. Zeng, and C. A. Grimes, "A wireless, passive carbon nanotube-based gas sensor," IEEE Sens. J., vol. 2, no. 2, pp. 82-88, Apr. 2002.

[16] S. Chopra, S. Natarajan, and A. M. Rao, "Gas sensing using carbon nanotube-based resonator," in Proc. IEEE Sens., Vienna, Austria, Oct. 2004, pp. 399-402.

[17] P. Russer, N. Fichtner, P. Lugli, W. Porod, J. A. Russer, and H. Yordanov, "Nanoelectronics-based integrated antennas," IEEE Microw. Mag., vol. 11, no. 7, pp. 58-71, Dec. 2010.

[18] A. Mehdipour, I. Rosca, A. Sebak, C. W. Trueman, and S. V. Hoa "Advanced carbon-fiber composite materials for RFID tag antenna applications," Appl. Comput. Electromagn. Soc. J. (Special Issue), vol. 25, no. 3, pt. Part I, pp. 218-229, Mar. 2010.

[19] Y. Zhou, Y. Bayram, F. Du, L. Dai, and J. Volakis, "Polymer-carbon nanotube sheets for conformal load bearing antennas," IEEE Trans. Antennas Propag., vol. 58, no. 7, pp. 2169-2175, Jul. 2010.

[20] G. Marrocco, L. Mattioni, and C. Calabrese, "Multi-port sensor RFIDs for wireless passive sensing-Basic theory and early simulations," IEEE Trans. Antennas Propag., vol. 56, no. 8, pp. 2691-2702, Aug. 2008.

[21] M. Meyyappan, Carbon Nanotubes Science and Applications. Boca Raton, FL: CRC, 2005.

[22] A. Mehdipour, I. Rosca, A. Sebak, C. W. Trueman, and S. V. Hoa, "Full-composite fractal antenna using carbon nanotubes FR multiband wireless applications," IEEE Antennas Wireless Propag. Lett., vol. 9, pp. 891-894, 2010

[23] S. J. Orfanidis, "Electromagnetic waves and antennas," Rutgers Univ., New Brunswick, NJ, 2008. [Online]. Available: http://www.ece.rutgers.edu/ rfanidi/ewa/

[24] P. V. Nikitin and K. V. S. Rao, "Theory and measurement of backscattering from RFID tags," IEEE Antennas Propag. Mag., vol. 48, no. 6, pp. 212-218, Dec. 2006.

[25] F. Fuschini, C. Piersanti, F. Paolazzi, and G. Falciasecca, "Analytical approach to the backscattering from UHF-RFID transponder," IEEE Antennas Wireless Propag. Lett., vol. 7, pp. 33-35, 2008.

[26] G. Marrocco, "RFID GRIDS: Part I-Electromagnetic theory," IEEE Trans. Antennas Propag, vol. 59, no. 3, pp. 1019-1026, Mar. 2011.

[27] S. Caizzone and G. Marrocco, "RFID GRIDS: Part II-Experimentations," IEEE Trans. Antennas Propag., vol. 59, no. 8, pp. 2896-2904, Aug. 2011.

[28] G. Marrocco, "The art of UHF RFID antenna design: Impedancematching and size reduction techniques," IEEE Antennas Propag. Mag., vol. 50, no. 1, pp. 66-79, Feb. 2008

[29] C. A. Desoer and E. S. Kuh, Basic Circuit Theory, F. Angeli, Ed. Italian ed. Milan, Italy: Franco Angeli Edizioni, 1990, pp. 441-441.

[30] Y. Lee, "Antenna circuit design for RFID applications," Microchip Technol. Inc., Chandler, AZ, Appl. Note AP7 10, 2003. [Online]. Available: http://www.microchip.com/downloads/en/AppNotes/00710c.pdf

[31] R. E. Collin, Antennas and Radiowave Propagation. New York: McGraw-Hill, 1985.

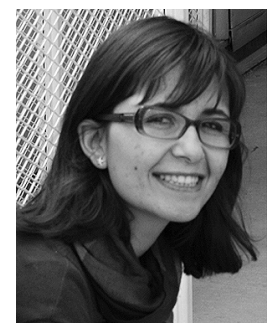

Cecilia Occhiuzzi (S'10-M'11) received the M.Sc. degree in medical engineering from the University of Rome "Tor Vergata," Rome, Italy, and is currently working toward the Ph.D. degree in electromagnetics (with an interest is wireless health monitoring by means of wearable and implantable RF identification techniques) at the University of Rome "Tor Vergata."

In 2008, she was with the School of Engineering, University of Warwick, Warwick, U.K., as a postgraduate student involved with design and implementation of wireless surface acoustic wave (SAW) sensors. In 2010, she was a Visiting Researcher with the Georgia Institute of Technology, Atlanta. She holds two patents on sensor RFID systems. Her research was mainly focused on the design of passive RFID sensors for structural health monitoring and gas detection by means of CNT-based tags. 


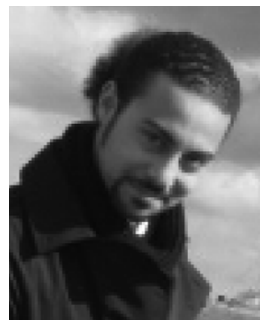

Amin Rida (S'05-M'10) received the B.S., M.S., and Ph.D. degrees in electrical and computer engineering from the Georgia Institute of Technology, Atlanta, in 2006, 2009, and 2011, respectively.

He coauthored RFID-Enabled Sensor Design and Applications (Artech House, 2010). He also coauthored two book chapters, seven journal papers, and 30 conference papers in peer-reviewed publications. He has six patents pending. His research interests include design, development, and packaging of electronics (antennas, interconnects, 3-D transitions) and their integration on organic, flexible, and high-performance substrates for millimeter-wave frequencies. His other research topics focus on inkjet printed electronics, characterization of organic substrates for RF applications, antenna design for RFID applications, development of wireless transceivers for sensing and power scavenging applications, $X$-band filter design and passive RF sensing such as CNT-based sensors and structural monitoring or smart skin applications.

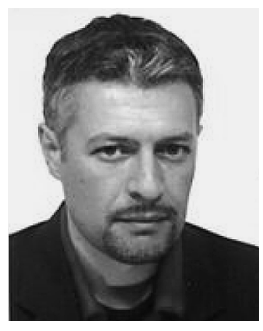

Gaetano Marrocco was born in Teramo, Italy, on August 29, 1969. He received the Laurea degree in electronic engineering (Laurea cum laude and Academic Honor) and Ph.D. degree in applied electromagnetics from the University of L'Aquila, L'Aquila, Italy, in 1994 and 1998, respectively.

Since 1997, he has been a Researcher with the University of Rome "Tor Vergata," Rome, Italy, where he currently teaches antenna design and medical radio-systems, manages the Antenna Laboratory, the RFID Laboratory and is Advisor in the Geo-Information Ph.D. program. In 2010, he became an Associate Professor of electromagnetic. In 1994, he was with the University of Illinois at Urbana-Champaign, as a Postgraduate Student. In 1999, he was a Visiting Researcher with Imperial College, London, U.K. In 2008, he joined the Ph.D. program of the University of Grenoble, Grenoble, France. His research is mainly directed to the modeling and design of broadband and ultra-wideband (UWB) antennas and arrays, as well as of sensor-oriented miniaturized antennas for biomedicine, aeronautics and RFID. He has been involved in several space, avionic, naval, and vehicular programs of the European Space Agency (ESA), North Atlantic Treaty Organization (NATO), Italian Space Agency, and the Italian Navy concerning the analysis and the design of nonconventional antennas and systems over platforms. He holds eight patents on broadband naval antennas and structural arrays and on sensor RFID systems.

Prof. Marrocco is currently an associate editor of the IEEE ANTENNAS AND Wireless PROPAGATION LeTters. He is as member of the Technical Program Committee of IEEE RFID, IEEE Microwave Theory and Techniques Society (IEEE MTT-S) International Microwave Symposium (IMS), and ISABEL. In 2008, he was the general chairman of the first Italian multidisciplinary scientific workshop on RFID: RFIDays-2008: Emerging Technology for Radiofrequency Identification. He was the co-chair of the RFIDays-2010 International Workshop held in Finland and chairman of the Local Committee of the V European Conference on Antennas and Propagation. In 2011, he was nominated vice-chair of the Italian delegation URSI Commission D: Electronics and Photonics.

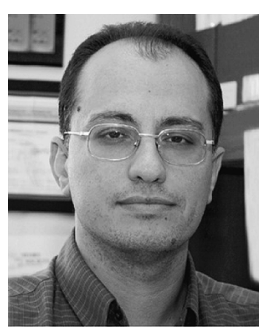

Manos Tentzeris (S'89-M'92-SM'03-F'10) received the Diploma degree (magna cum laude) in electrical and computer engineering from the National Technical University of Athens, Athens, Greece, and the M.S. and Ph.D. degrees in electrical engineering and computer science from The University of Michigan at Ann Arbor.

He is currently a Professor with the School of Electrical and Computer Engineering, Georgia Institute of Technology, Atlanta. He has authored or coauthored over 370 papers in refereed journals and conference proceedings, five books, and 19 book chapters. He has helped develop academic programs in highly integrated/multilayer packaging for RF and wireless applications using ceramic and organic flexible materials, paper-based RFIDs and sensors, "green" electronics and power scavenging, nanotechnology applications in RF, microwave microelectromechanical systems (MEMS), system-on-package (SOP)-integrated (ultra-wideband (UWB), multiband, conformal) antennas and adaptive numerical electromagnetics (FDTD multiresolution algorithms), and heads the ATHENA Research Group (20 researchers). He is an Associate Editor for the International Journal on Antennas and Propagation.

Dr. Tentzeris is a member of URSI Commission D and the MTT-15 Committee. He is an associate member of the European Microwave Association (EuMA). He is a Fellow of the Electromagnetic Academy. He is a member of the Technical Chamber of Greece. He is an IEEE Microwave Theory and Techniques Society (IEEE MTT-S) Distinguished Microwave Lecturer (2010-2012). $\mathrm{He}$ is an associate editor for the IEEE TRANSACTIONS ON MICROWAVE THEORY AND TECHNIQUES and the IEEE TRANSACTIONS ON ADVANCED PACKAGING. 